\title{
Intervarietal Differences in Karyotype of Tea
}

\author{
Madhuparna Datta and Beena Agarwal \\ National Agricultural and Scientific Research Foundation, \\ 113/2, Amherst Street, Calcutta 700 009, India
}

Accepted April 13, 1992

Tea Camellia sinensis (L.) O. Kuntze is one of the most important cultivated crop plant of India. The genomic account of this plant have become very much complicated due to natural hybridization (Wight and Barua 1957, Wood and Barua 1958, Bezbaruah 1975). Though several authors (Ackerman 1971, 1973, Bezbaruah 1977, Chaudhuri and Bezbaruah 1985) worked on the genomic set up at various ploidy level of this plant, there is no report on detailed karyotype study at varietal level. The present work deals with detail karyotype analysis to find out the intervarietal relationship among 6 diploid clonal cultivars (Tocklai Vegetative [TV] variety) extensively grown in Northeast India. As the clonal varieties are mainly based on yield and quality, our another object is to see whether there is any interrelation among yield, quality and karyotype at the varietal level.

\section{Materials and methods}

Fresh shoot tips from mother bush of 6 clonal varieties- $T V_{1}, T_{19}, T_{20} T_{21}, T V_{22}$ and TS 491 were collected from Assam tea garden.

Well scattered metaphase plates were obtained by pretreating the shoot tips with paradichlorobenzene, $0.002 \mathrm{M}$ Hydroxyquinolene $(1: 1)$ and aesculine for $41 / 2 \mathrm{hr}$. followed by overnight fixation in 3:1 ethanol acetic acid mixture. The best staining was obtained in $2 \%$ aceto-orcein after a prior hydrolysis in $5 \mathrm{~N} \mathrm{HCl}$ for $8 \mathrm{~min}$. in $0-4^{\circ} \mathrm{C}$.

The individual chromosome volume was calculated by the formula $\pi r^{2} h$ where $r$ is the radius and $\mathrm{h}$ is the length of the chromosome. The total chromosome length and volume with standard error (S. E.) were computed by adding the individual length and volume respectively. The analysis of variance (ANOVA) at the intervarietal level was also calculated. Typification of chromosomes has been done on the basis of centromere position.

\section{Observations}

The somatic chromosome number $(2 n=30)$ and number of nucleolar chromosomes were found constant in 6 varieties. Though a gross morphological similarity was revealed in all the varieties, the number of different chromosome types varied to a significant level (Table 1). Chromosomes were mostly of medium size $(2-5 \mu \mathrm{m})$, but one pair of small chromosomes was noted in all varieties. All the chromosomes were divided into 6 types of which 2 types were designated as the nucleolar chromosomes:

Type A: Nucleolar chromosome with nearly subterminal and nearly median constrictions. Range of length was in between 2.41-4.07 $\mu \mathrm{m}$.

Type B: Nucleolar, with nearly subterminal and nearly submedian constrictions. The length range was $2.22-3.52 \mu \mathrm{m}$.

Type C: Medium size chromosomes $(1.48-5.0 \mu \mathrm{m})$ with nearly median primary constrictions. Type D: Medium to short size chromosomes $(1.11-3.59 \mu \mathrm{m})$ with nearly submedian constrictions. 
Type E: Medium (2.04-3.12 $\mu \mathrm{m})$ with nearly subterminal chromosomes.

Type F: Medium (1.48-2.96 $\mu \mathrm{m})$ with submedian primary constrictions.

A clear numerical and structural alterations of centromeric chromosomes were noted in all the studied varieties (Fig. 1). Chromosomes of ' $A$ ' type were found in $T V_{1}, T_{19}, T_{20}$

Table 1. Detailed description of chromosome types in 6 different varieties

\begin{tabular}{lcccl}
\hline \hline Variety & $\begin{array}{c}\text { Chromosome } \\
\text { No. }(2 \mathrm{n})\end{array}$ & $\begin{array}{c}\text { Range of chromo- } \\
\text { some length }(\mu \mathrm{m})\end{array}$ & $\begin{array}{c}\text { No. of nucleolar } \\
\text { chromosomes }\end{array}$ & $\begin{array}{c}\text { No. of different } \\
\text { chromosome types }\end{array}$ \\
\hline $\mathrm{TV}_{1}$ & 30 & $1.11-3.15$ & 2 & 2A 6C 20D 2E \\
$\mathrm{TV}_{19}$ & 30 & $1.48-4.07$ & 2 & 2A 16C 10D 2F \\
$\mathrm{TV}_{20}$ & 30 & $1.48-4.07$ & 2 & 2A 8C 18D 2F \\
$\mathrm{TV}_{21}$ & 30 & $1.11-2.96$ & 2 & 2B 10C 14D 2E 2F \\
$\mathrm{TV}_{22}$ & 30 & $1.48-4.44$ & 2 & 2B 16C 8D 2E 2F \\
$\mathrm{TS}_{491}$ & 30 & $1.11-2.96$ & 2 & 2A 16C 12D \\
\hline
\end{tabular}

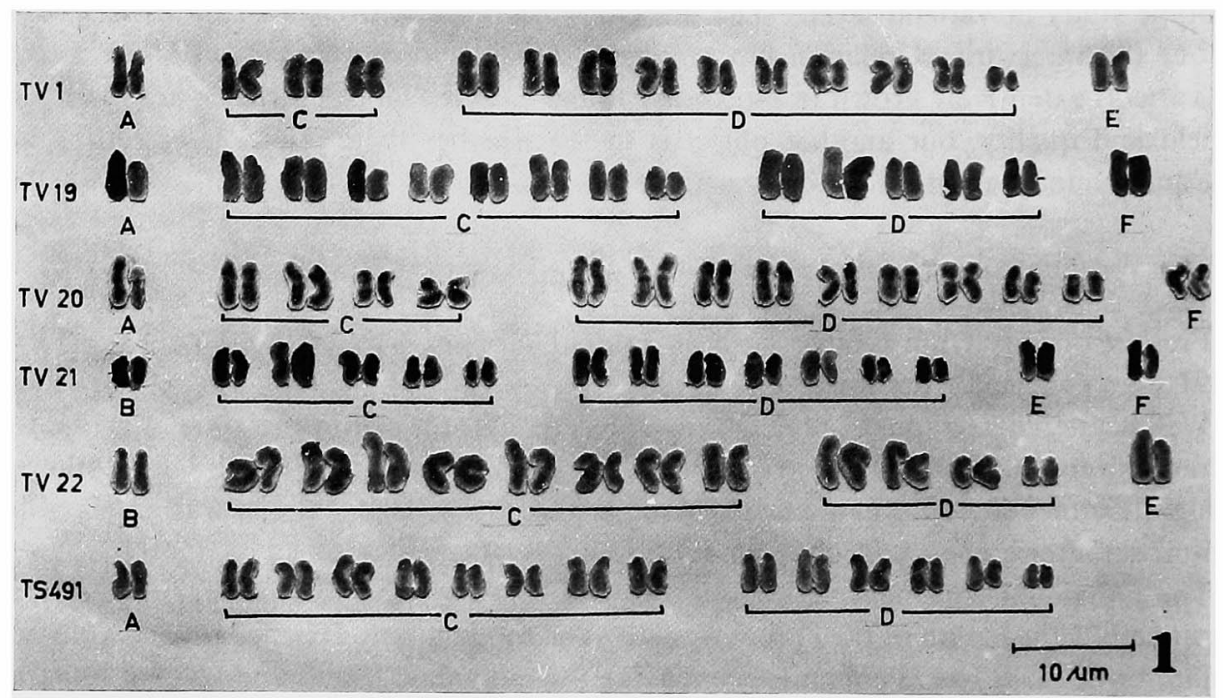

Fig. 1. Karyogram of 6 clonal varieties of tea showing number of different types of chromosomes at a glance.

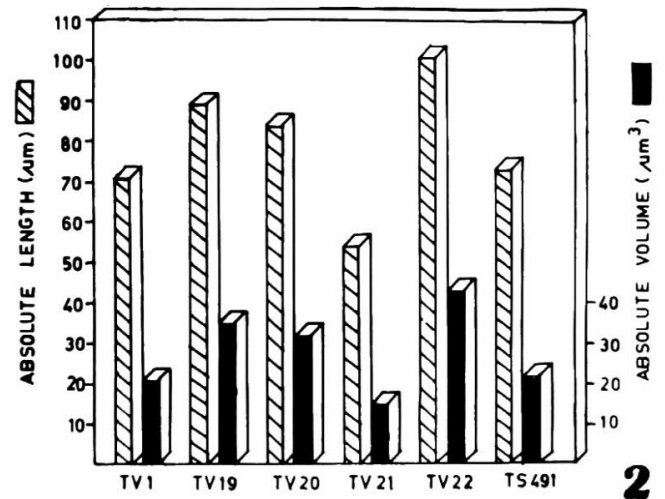

Fig. 2. A comparative account of absolute genomic length and volume in 6 varieties represented by histogram. and TS 491 but absent in $\mathrm{TV}_{21}$ and $\mathrm{TV}_{22}$ where ' $B$ ' type present. ' $C$ ' and ' $D$ ' type of chromosomes were found to present in all varieties but number of each type was different (Table 1, Fig. 1). 'E' type was found to be present in 3 varieties $\left(\mathrm{TV}_{21}, \mathrm{TV}_{1}\right.$ and $\mathrm{TV}_{22}$ ) and ' $\mathrm{F}$ ' type was found in $\mathrm{TV}_{21}, \mathrm{TV}_{20}$, $\mathrm{TV}_{19}$ and $\mathrm{TV}_{22}$. Both ' $\mathrm{E}$ ' and ' $\mathrm{F}$ ' types were absent in TS 491 (Fig. 1). The overall observations revealed asymmetric karyotypes in all the studied varieties which was reflected in total $\mathrm{F} \%$. However, the total $\mathrm{F} \%$ did not show any marked variation (Table 2 ).

Though the number was same, the absolute length and volume of total genomic 


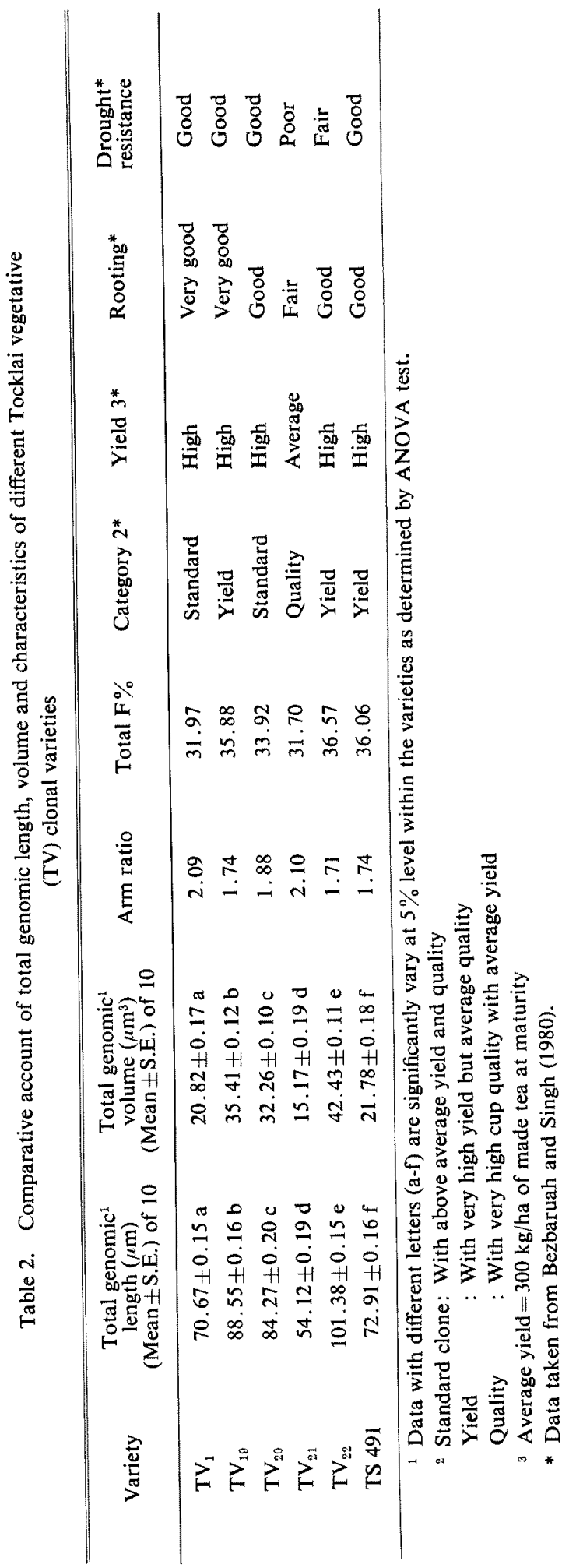


constituents differed within the varieties (Table 2). The highest values were observed in $\operatorname{TV}_{22}\left(101.38 \pm 0.15 \mu \mathrm{m} ; 42.43 \pm 0.11 \mu \mathrm{m}^{3}\right)$ whereas the lowest in $\operatorname{TV}_{z .1}(54.12 \pm 0.19 \mu \mathrm{m}$; $15.17 \pm 0.19 \mu \mathrm{m}^{3}$ ). The variation was significant in other varieties also (Table 2, Fig. 2). The characteristics of different varieties as depicted in Table 2, were taken from Bezbaruah and Singh (1980).

\section{Discussions}

The overall observations reveal a number of important findings. Though the chromosome number is same, karyotype analyses show clear differences among the varieties. Variation in nucleolar chromosome type is also observed (Table 1). The number of different chromosome types is also differed (Fig. 1, Table 1). Total F\% and arm ratio proves asymmetry in all karyotypes studied (Table 2). The minute changes in chromosome types at the varietal level may be account for changes in heterochromatic part of the genome.

$\mathrm{TV}_{1}$ being the first clonal variety (Bezbaruah and Singh 1980) shows lower genomic length and volume than the later developed varieties like $T V_{18}, T V_{20}$ and $T V_{22}$. But both length and volume are lowest in $\mathrm{TV}_{21}$ (Table 2, Fig. 2). Notwithstanding the fact that chromosome length and volume are greatly dependent on differential spiralization, despiralization and histone protein content at the individual chromosome level (Mukherjee and Sharma 1986, Mukhopadhyay and Sharma 1987, Das and Mallick 1989), a clear correlation between the genomic length and volume is found to be significant in this study (Table 2). The increase in total genomic length is associated with the increase in volume. The differences within the varieties are also found significant by ANOVA test.

With a view to evaluate the cytological parameters in respect to the characteristics of Tocklai varieties (Bezbaruah and Singh 1980), it is interesting that $\mathrm{TV}_{21}$ is quite indifferent in having the lowest genomic length, volume and highest arm ratio. While the other varieties are of standard or yield category, $\mathrm{TV}_{21}$ is the only quality variety having average yield, fair rooting and poor drought resistance (Table 2). Though it is not possible to correlate these apparently unrelated characters in the light of present findings, but the intervarietal variation is significant as their manifestation is clearly controlled at the genic level.

\section{Summary}

Detailed karyotype analyses of 6 cultivars of tea vegetative clones are done. On the basis of centromere position, chromosomes are classified into 6 types. Number of different types varied within the varieties. Both ' $E$ ' and ' $F$ ' type are absent in TS 491. Total genomic length and volume are also considered as the parameters. Singnificant variation is observed at the varietal level. $\mathrm{TV}_{21}$, a quality clone, is found quite indifferent in karyotype, total length and volume of the chromosomes. The other characteristic features of this variety is also different from the rest of varieties. The overall study reveals a clear varietal demarcation.

\section{References}

Ackerman, W. L. 1971. Genetic and cytological studies with Camellia and related genera. Technical Bulletin No. 1427. USDA.

- 1973. Aneuploidy in Camellia. J. Hered. 64: 197-202.

Bezbaruah, H. P. 1975. Cytological investigation in the family Theaceae. II. Cytology of a triploid tea. Exp. Agric. 11: 13-16.

- 1977. Aneuploidy in tea. The Nucleus 19: 167-169.

— and Singh, I. D. 1980. Characteristics of Tocklai released planting materials. Two and A Bud. 27 (1): 6-8. 
Chaudhuri, T. C. and Bezbaruah, H. P. 1985. Morphology and anatomy of aneuploid and polyploid tea [Camellia sinensis (L) O. Kuntze]. J. Plantation Crops 13 (1): 22-30.

Das, A. B. and Mallick, R. 1989. Variation in karyotype and nuclear DNA content in different varieties of Foeniculum vulgare Mill. Cytologia 54: 129-134.

Mukherjee, S. and Sharma, A. K. 1986. Intervarietal relationship between chromosome size, nuclear DNA amount and protein content in Cajanus cajan (L.) Mill sp. Perspectives in Cytology and Genetics (Manna, G. K. and Sinha, U. eds.) 5: 735-740.

Mukhopadhyay, S. and Sharma, A. K. 1987. Karyomorphological analysis of different species and varieties of Calathea, Maranta and Stromanthe of Marantaceae. Cytologia 52: 821-831.

Wight, W. and Barua, P. K. 1957. What is tea? Nature 179: 506-507.

Wood, D. J. and Barua, P. K. 1958. Species hybrids of tea. Nature (Lond) 181 : 1674-1675. 